\title{
Presentación de la Teoría Social de la Comunicación
}

\author{
Manuel MARTIN SERRANO
}

REFERENCIA PARA LAS CITAS DE ESTA PUBLICACIÓN Y DE SUS CONTENIDOS:

MARTIN SERRANO, Manuel (2004): "Presentación de la Teoría Social de la Comunicación", en La producción social de comunicación. Madrid: Alianza (3 $3^{\text {a }}$ edición revisada; 1aㅡ edición 1986 y 2ª edición revisada 1993), pp. 11-34. ISBN: 84-206-4192-8.

Recuperado el_de de 2 de http://eprints.ucm.es/13237/

\section{UTILIZACIÓN DE ESTE DEPÓSITO:}

Usted es libre de copiar, distribuir y comunicar públicamente la obra bajo las siguientes condiciones, que corresponden a la licencia Creative Commons que protege este texto:

Reconocimiento. Debe reconocer y citar al autor original, utilizando la "REFERENCIA PARA LAS CITAS DE ESTA PUBLICACION Y DE SUS CONTENIDOS" (véase recuadro superior).

No comercial. No puede utilizar esta obra para fines comerciales.

Sin obras derivadas. No se puede alterar, transformar, o generar una obra derivada a partir de esta obra. 


\title{
Reading LA PRODUCCIÓN SOCIAL DE COMUNICACIÓN. LA COMUNICACIÓN PÚBLICA EN EL CAMBIO DEL MUNDO
}

\author{
Presentación y estudio documental por Daniel Franco Romo
}

En E-Prints se tiene acceso a una selección de la obra original de Manuel Martín Serrano (véase: "Publicaciones de Manuel Martín Serrano disponibles en E-Prints. Selección sistematizada"*, en http://eprints.ucm.es/11107/).

Una parte importante de dicha obra está dedicada al estudio de LA PRODUCCIÓN SOCIAL DE COMUNICACIÓN. La producción social de comunicación (Madrid: Alianza, 1986, 1993, 2004) es un libro que integra teoría, metodología e investigación y con el que Manuel Martín Serrano contribuye decisivamente a que la comunicación forme parte de las ciencias sociales. Esta obra ofrece una perspectiva y una sistemática específicas, para investigar las relaciones entre sociedad y comunicación. Introduce, como un componente necesario para el análisis de los cambios históricos de las sociedades, las sucesivas transformaciones de la comunicación pública, en sus diversos niveles: transformaciones de las tecnologías, de las organizaciones que tienen a su cargo la labor de proveer de información a la colectividad, y de los usos que de todo ello se hace en cada comunidad. La producción social de comunicación es un título de referencia en la bibliografía científica de la comunicación. Su influencia se refleja en el acervo de publicaciones que se han distanciado de la "teoría de la comunicación social" para trabajar con el enfoque de una "teoría social de la comunicación". Es evidente el giro que representa este cambio semántico.

En este Reading, se han seleccionado textos que corresponden a los tres niveles en los que se mueve esta parte de la obra del autor: el teórico, el de los usos sociales de la comunicación, el de los desarrollos metodológicos.

\section{Creación de la Teoría Social de la Comunicación}

Manuel Martín Serrano escribe que la producción social de comunicación es una actividad sociohistórica que requiere enfoques macrosociológicos. En consecuencia, propone e inicia la Teoría Social de la Comunicación. Está descrita en este depósito: "Presentación de la Teoría Social de la Comunicación". Los cambios que se están produciendo en el uso y en el valor de la información requieren este desarrollo teórico. El estudio de por qué y cómo hay que llevar a cabo esa renovación teórica está en uno de los artículos más esclarecedores del autor: "La epistemología de la comunicación a los cuarenta años de su nacimiento" (http://eprints.ucm.es/13238/).

\section{Orígenes y características de los usos actuales de la comunicación pública}

Cuando Manuel Martín Serrano elabora la Teoría Social de la Comunicación, ya había investigado y publicado sobre los formas históricas de producir comunicación pública y sus usos sociales, desde la Modernidad hasta la época actual. El tránsito desde la comunicación en la sociedad postindustrial a la comunicación en la emergente sociedad globalizada se describe en La mediación social (Madrid: Akal, 1977, 2008). Entre los escritos en los que se ha referido a ese tránsito, se ha seleccionado "Las transformaciones sociales vinculadas a la era audiovisual" (http://eprints.ucm.es/13187/) y "La ampliación de la realidad en la que vivimos con otro universo virtual" (http://eprints.ucm.es/11069/).

A comienzos de los años 80, anticipa que "La nueva era no va a ser de la comunicación, sino la de la conexión" (http://eprints.ucm.es/11065/). El autor reanaliza en clave de economía política las transformaciones en la producción comunicativa, tal como se puede apreciar en "Cuándo el valor de cambio de la información puede ser medido" (http://eprints.ucm.es/11067/). Y, en clave prospectiva, muestra que los mismos factores que están ampliando las aplicaciones sociales de las tecnologías comunicativas, también tienen un enorme potencial de desorganización. El análisis de cómo se verá afectado el actual orden de monopolio globalizado está resumido en "La forma vigente de producir comunicación pública. Desarrollo y quiebra" (http://eprints.ucm.es/13239/). El artículo publicado en Reis "Los cambios acontecidos en las funciones de la comunicación y en el valor de la información" (http://eprints.ucm.es/13240/) relaciona la revolución informático-comunicativa con las transformaciones históricas que están en curso. La utilización de las nuevas formas de producir comunicación acabará remodelando, a escala universal, las formas de acción social. 
Manuel Martín Serrano se ha referido en varias ocasiones a la naturaleza dual que es característica de la producción social de comunicación: innovadora y redundante, controladora y al tiempo liberadora. Cree que por primera vez existen capacidades para que pueda decantarse hacia el desarrollo de la creatividad y la autonomía individuales. Véase en el texto "La gran paradoja que presenta la evolución histórica de la comunicación pública" (http://eprints.ucm.es/11063/).

\section{La producción social de comunicación y el colonialismo cultural}

La comunicación pública ha contribuido y lo sigue haciendo al colonialismo, desde la época de los descubrimientos a la de la globalización. Manuel Martín Serrano indica que ese uso la convierte en un arma de guerra y en un procedimiento de explotación. El autor escribe con frecuencia artículos sobre este tema, desde la perspectiva teórica e histórica que distingue a su obra. "Orígenes históricos de los usos actuales de la comunicación pública" (http://eprints.ucm.es/13242/) regresa a la época en la que los movimientos religiosos inventaron "la comunicación social" (a finales del siglo XIX) como herramienta para la catequesis. El neocolonialismo se apropia ese mismo modelo manipulador y lo adapta para crear dependencia. Aclara el autor que "el colonialismo se convierte en neocolonialismo cuando logra integrar el comercio y la transculturización en una única red". En nuestra época de producción en masa de estereotipia, el neocolonialismo tiene a todas las naciones por su territorio. Produce "La comunicación que globaliza la pobreza cultural" (http://eprints.ucm.es/13244/). Empobrecimiento que lleva implícito la sustitución del recurso a cualquier lenguaje hablado por la acción: véase "Cuando la eliminación del idioma propio hace de «la acción» el modo de narrar único o principal” (http://eprints.ucm.es/13245/). Manuel Martín Serrano ha acuñado categorías para describir la forma en la que se produce esa destrucción de bienes intangibles, en "La comunicación pública y la supervivencia" (http://eprints.ucm.es/13246/). También se ha seleccionado un texto que contiene, en su brevedad, importantes enseñanzas para el estudio y el entendimiento de cómo funcionan esos mecanismos de sometimiento: "El colonialismo cultural se analiza investigando las relaciones entre acción y comunicación" (http://eprints.ucm.es/13247/). Explica que la desorganización de las instituciones productivas, familiares y políticas de una comunidad es condición necesaria para que la producción comunicativa que transculturiza quebrante los valores y transforme las costumbres.

\section{Metodologías y técnicas para investigar la producción social de comunicación}

Manuel Martín Serrano suele elaborar los modelos de investigación que se adecuan a los planteamientos teóricos que él mismo ha propuesto. Por ejemplo, crea en La producción social de comunicación los conocidos conceptos de "Mediación cognitiva y estructural" (http://eprints.ucm.es/13166/). Son desarrollos de la teoría de la mediación, para el estudio de la comunicación pública, que se acompañan en dicho libro de los correspondientes "Diseños para investigar la producción social de comunicación" (http://eprints.ucm.es/13147/). Se reproduce "Un protocolo para llevar a cabo estudios paradigmáticos de la producción social de comunicación" (http://eprints.ucm.es/13248/), que se viene utilizando ininterrumpidamente, por su autor y por otros muchos investigadores.

\section{REFERENCIAS para enlazar este documento con los que cita y con aquellos que le citan}

\section{- “Prólogo para La mediación social en la era de la globalización” (http://eprints.ucm.es/10651/)}

- "La comunicación NO es una alternativa autónoma respecto al sistema de producción y de reproducción social" (http://eprints.ucm.es/11049/)

- “Las tres formas de empleo de la comunicación” (http://eprints.ucm.es/11058/)

- "La gesta y la parábola en los relatos de la comunicación pública” (http://eprints.ucm.es/11061/)

- "Innovación tecnológica, cambio social y control social” (http://eprints.ucm.es/11064/)

- "Mitos y carencias" (http://eprints.ucm.es/11066/) 
- "La transferencia de la ejecución a la indicación en los comportamientos humanos" (http://eprints.ucm.es/13105/)

- “Las relaciones macrosociológicas entre acción y comunicación” (http://eprints.ucm.es/13107/)

- "La comunicación en la existencia de la humanidad y de sus sociedades" (http://eprints.ucm.es/13112/)

- "El modelo dialéctico de la comunicación" (http://eprints.ucm.es/13116/)

- “Para qué sirve estudiar Teoría de la Comunicación?” (http://eprints.ucm.es/13145/)

- “Dialéctica, comunicación, mediación” (http://eprints.ucm.es/13254/)

- “La Teoría de la Comunicación, la vida y la sociedad" (http://eprints.ucm.es/13109/)

- “CIESPAL y la humanización de la comunicación: Puente entre el estado de las ciencias y la práctica de la comunicación” (http://eprints.ucm.es/13183/).

*Esta selección y sistematización de publicaciones de Manuel Martín Serrano, así como los análisis que les acompañan, se basa principalmente en los estudios realizados por los especialistas que han participado en dos monográficos dedicados a la obra del autor: el primero editado por Anthropos y preparado por Esteban Mate y el segundo por Chasqui, coordinado por Francisco Bernete. También se han localizado y utilizado numerosas reseñas que están publicadas en otras revistas científicas. El investigador Daniel Franco Romo ha planificado y supervisado la ejecución de todo el proyecto. 


\title{
Presentación de la Teoría Social de la Comunicación
}

\author{
Manuel MARTIN SERRANO
}

Este libro trata de la producción de información destinada a la comunicación pública y de su uso social. Se interesa por las características de las sucesivas modalidades de comunicación pública que existen y que han existido. Se ocupa del origen y de las transformaciones de los Sistemas Institucionales de Comunicación, encargados en cada sociedad de la elaboración y distribución de las noticias que conciernen al conjunto de la colectividad. Identifica los diversos componentes tecnológicos, organizativos y culturales que incluye cada Sistema Institucional de Comunicación. Estudia cómo se producen una clase de bienes fabricados para abastecer a la comunidad de información: los productos comunicativos. Analiza el uso que se hace de esa información para contribuir a la producción y reproducción de la sociedad. Pone en relación las peculiaridades materiales y narrativas de los productos comunicativos con las funciones que cumplen en cada sociedad.

La Producción Social de Comunicación ofrece una perspectiva y una sistemática específicas para investigar las relaciones entre sociedad y comunicación. Ha iniciado un nuevo campo de estudios cuyas aportaciones también son útiles para otras ciencias sociales .He propuesto que la naciente ciencia se denomine «Teoría Social de la Comunicación».

La Teoría Social de la Comunicación, como toda otra, funda su existencia en algún supuesto. El único que se da por establecido es el siguiente: existen interdependencias entre la transformación de la comunicación pública y el cambio de la sociedad. El estudio de esas relaciones puede ser elevado al estatuto científico de una teoría. Para ello se requiere un trabajo heurístico, metodológico y de investigación importante. Hay que identificar la naturaleza de esas mutuas afectaciones. Formular las leyes históricas que permitan explicar y predecir cuándo, por qué y cómo se producen. Encontrar criterios que permitan prever los efectos que tienen tales interacciones para el progreso o el estancamiento de los hombres; sobre su cultura, sus relaciones y sus instituciones.

La producción social de comunicación está escrita para definir esta teoría y fundar sus cimientos. Consciente de que estoy explorando un territorio demasiado vasto para mi tiempo y mis solas capacidades, mi trabajo quiere proporcionar la simiente de nuevas ideas y la herramienta de nuevas metodologías. Por eso he asumido el riesgo del error científico pero no el de la banalidad. Un libro de creación y no de recreación, precisamente para serlo, obliga a poseer un buen conocimiento de la obra de otros; sobre todo la obra de quienes tenemos por clásicos de las Ciencias Sociales porque innovaron sus paradigmas teóricos. He tratado de recuperar para la Teoría Social de la Comunicación las aportaciones de esos autores, situando sus textos en este otro contexto. Ese es, tal vez, el mejor aprecio que cabe hacer del pensamiento ajeno. El mío les debe un marco intelectual, un lenguaje y un aura en el que se conformó la visión del saber entre las personas de mi generación. Pero mi cita con la creación teórica se fijó en otro lugar menos frecuentado de la ciencia social donde se cruzan los caminos epistemológicos de las ciencias del conocimiento y de la historia de las ideas con los estudios del cambio y de la reproducción de las sociedades. Para llegar a ese punto de encuentro una parte importante de mi afán ha transcurrido por los espacios teóricos de la comunicación. ${ }^{1}$

\footnotetext{
${ }^{1}$ Existen en la Semiología desde Pierce, en la Teoría General de la Comunicación desde Shannon y Beaver y en el Análisis de Sistemas desde von Bertalanffy herramientas conceptuales a las que convenía que se abriesen las ciencias sociológicas, lastradas por el uso descriptivo de la estadística y el hermenéutico de la Psicología Dinámica. Las correspondientes metodologías me pareció que podían ser integradas como variantes de modelos lógicos, los cuales permitirían procedimientos de diseño, de cálculo y de verificación más universales. Esa fue mi primera
} 
Este libro no podría haber sido escrito antes de que existiese su propio paradigma, es decir una concepción de la naturaleza de la comunicación que sirva de soporte teórico al conjunto de las ciencias que estudian tales interacciones. Esa reflexión previa está publicada en otros libros que se irán mencionando cuando sea pertinente. En la medida que La producción social de comunicación se asiente en un paradigma sólido, cumplirá con el propósito de ser un texto para iniciar una nueva ciencia social y no un mero libro introductorio a los fenómenos de la comunicación pública. Con ese objetivo este libro propone el repertorio de leyes y de categorías que me parecen necesarias para sustentar a una Teoría Social de la Comunicación y desarrolla unas metodologías que permiten estudiar los objetos que le son propios. Para verificar la potencia analítica y la validez científica de los conceptos, se requiere de una investigación específica que permita probar las categorías teóricas y las metodologías. Dicha investigación está recogida en la primera edición de esta obra (Madrid. Alianza Universidad. AUT 102, 1986).

Esta introducción explica por qué el libro es como está hecho; es decir, la relación que existe entre la estructura del libro el objeto de que trata y el objetivo científico que se pretende.

\section{NIVEL EN EL QUE SE SITÚA EL ESTUDIO CIENTIFICO DE LA PRODUCCIÓN SOCIAL DE COMUNICACIÓN}

La producción social de comunicación es punto de partida para estudiar las relaciones que existen entre la transformación de la comunicación pública y el cambio de las sociedades. Como este tema delimita un nuevo ámbito teorético en el campo de las Ciencias Sociales requiere de un planteamiento paradigmático.

El enfoque paradigmático se distingue porque no se limita a describir una actividad; en este caso la producción, distribución y uso de la información que concierne a la comunidad. Además los datos se utilizan para probar la pertinencia de alguna teoría y para desarrollar metódicamente una herramienta heurística. ${ }^{2}$

El científico puede trabajar a este nivel teórico sólo en contadas ocasiones, porque los objetos de investigación adecuados son escasos ${ }^{3}$. Los objetos pertinentes para producir teoría tienen que ser prácticas sociales tan universales como sea posible. Por ejemplo, el desear, el razonar, el constreñir. Así, la Economía Política le ofreció su espejo a Marx para que, en los procesos referidos al cambio de valores, comprobase lo que cabía esperar de la aplicación de la dialéctica a la historia; la Psiquiatría fue el banco de pruebas utilizado por Jaspers para incorporar la fenomenología al campo de las Ciencias Humanas, entrando en ellas por el análisis de la enfermedad mental; la Psicología Genética ha sido el terreno en el que Piaget transformó el paradigma estructuralista en una herramienta apta para el estudio de los procesos cognitivos.

\footnotetext{
aportación desgranada durante seis años de trabajo heurística en sucesivas publicaciones entre ellas "Aplicación de la teoría y el método sistemático en ciencias sociales”, "Epistemología del Realismo Sociológico”, "Epistemología de la dialéctica social”, “Un método lógico para analizar el significado”, "Métodos actuales de investigación social”. (Cf. en la bibliografía que incluye este libro). La elaboración sistemática de estos planteamientos concluye para mí con la propuesta de la Teoría de la Mediación, (Cf. La mediación social). Mi primera entrada en el campo de la comunicación pública se produjo en ese mismo tiempo y tuvo por objeto probar el funcionamiento del nuevo paradigma, en "Análisis de la Visión del Mundo en la TV” y en "L'Ordre du monde a travers la TV”. En ambas obras están configurados los conceptos y los métodos de trabajo que se pueden reconocer en este libro.

${ }^{2}$ Una teoría llega a ser referencia científica para un campo del saber después de que se haya tomado a sí misma como objeto de conocimiento. Ese paso se franquea observando el modo de observar que la Teoría adopta cuando explica la naturaleza de alguna actividad concreta.

${ }^{3}$ Las prácticas sociales permiten que en ellas se pruebe la potencia explicativa de las teorías y de los métodos de investigación sólo durante el tiempo en el que todavía conservan su virginidad epistemológica. Es decir en tanto que su sentido no ha sido esclarecido y mientras que el conocimiento no esté irreversiblemente mistificado por los modelos del saber instrumental.
} 
Pocas actividades humanas, suficientemente universales y significativas, aguardan todavía a los nuevos Cook de las Ciencias Sociales amantes de la aventura intelectual que supone rellenar un espacio teórico vacío ${ }^{4}$. No obstante quedan todavía algunos fenómenos sociales sobre los que se saben muchas cosas pero aún se comprenden muy pocas. Uno de esos objetos es la comunicación institucionalizada. Las Ciencias de la Comunicación no cuentan con una teoría de la producción social de información que satisfaga requisitos epistemológicos equivalentes a los que ya cumplen las teorías cognitivas para las Ciencias de la mente, o las teorías de las necesidades para las Ciencias del comportamiento. El científico que tenga la comezón del descubrimiento teórico tiene en el campo de la comunicación pública una oportunidad de aportar algo nuevo al saber científico y no sólo al conocimiento instrumental de los procesos comunicativos ${ }^{5}$.

\section{SITUACIÓN DE LA QUE SE PARTE AL INICIAR LA TEORÍA SOCIAL DE LA COMUNICACIÓN}

\subsection{Sociedades en las que puede estudiarse la producción social de comunicación}

La información destinada al conjunto de la comunidad es un fenómeno de producción social a partir del momento en el que se institucionaliza el tratamiento y el uso de la comunicación pública. Esa institucionalización se logra creando una organización más o menos compleja especializada en esa tarea, a la que se le asignan recursos materiales y humanos. Estas acotaciones se van a desarrollar con el debido detalle en este libro (Cf. pp. 71 y sigs.). Las mutuas afectaciones existentes entre el cambio de la sociedad y la transformación de la comunicación podrán observarse en todas las comunidades del pasado y del presente que hayan institucionalizado alguna forma de operar con la información de interés común. Por tanto, el campo de estudio abarca desde algunas sociedades todavía primitivas hasta las más desarrolladas.

\footnotetext{
${ }^{4}$ Todos quienes hemos llegado al trabajo científico después de Marx, Jaspers, Piaget y de Freud, Darwin o LeviStrauss, estamos en una situación más desventajosa que ellos, desde el punto de vista de lo que puede esperar de nosotros la historia acumulativa del saber. La inferioridad de nuestras generaciones no es ni de genio -puesto que en algún lugar habrá nacido o nacerá quien intelectualmente pueda comparárseles- ni de conocimiento -puesto que gracias a la obra de todos quienes nos han antecedido, sabemos más de lo que ellos sabían-. El problema que limita la creatividad epistemológica de los científicos sociales de nuestra época surge por la escasez creciente de espacios intelectuales vírgenes. ¿Cómo se puede ya reflexionar sobre la necesidad humana al margen de las categorías psicoanalíticas, teorizar sobre la reproducción social ignorando el modelo marxista, interpretar el significado de los rituales sin tomar en cuenta los análisis funcionalistas, discurrir sobre la función del parentesco sin el cuadro de referencia de la antropología estructural? El psicoanálisis, la fenomenología, la dialéctica, el funcionalismo y el estructuralismo han encauzado la concepción científica del desear, del enfermar, del producir, del socializar, del comprender Sin duda las cartas para navegar en el océano de los comportamientos de los hombres tienen aún muchas coordenadas que corregir, y será útil volver a transitar por los mismos parajes que recorrieron los Padres Fundadores, ahora con mejores instrumentos y mayor sistemática. Pero en el mundo de las prácticas sociales apenas quedan tierras ignotas. Parece que a las Ciencias Humanas también les ha llegado ese momento histórico, tal vez más productivo pero seguramente menos apasionante, en el que los colonizadores de los espacios científicos van a sustituir a los descubridores.

${ }^{5}$ La escasa producción teórica referida a la comunicación pública es en gran parte el subproducto de investigaciones interesadas en el uso político y comercial de la información. Las generalizaciones sobre la naturaleza social de la comunicación que se aventuran en algunos de esos trabajos tienen el valor de elucubraciones más o menos atinadas. Los más burdos, aunque no los menos famosos, no soportan el contraste con las reglas del método científico. Cometen errores de diseño que afectan a la fiabilidad ya la significación de los datos: utilizan muestras insuficientes o sesgadas, aplican escalas o indicadores mal dimensionados, no controlan el efecto de las variables intervinientes, etc. Casi todos carecen de los dos requisitos indispensables para que una investigación pueda tener un uso científico: el primero que las hipótesis y las conclusiones remitan a los datos que las apoyan; y el segundo que esos datos se presenten de manera que puedan ser desmentidos por otras investigaciones (en términos técnicos, que las conclusiones puedan ser falseadas). Pero sobre todo no existe el enfoque teórico que lleva a relacionar metódicamente esas observaciones sobre las prácticas de la comunicación pública, con las peculiaridades que diferencian a las sociedades donde se producen.
} 


\subsection{El axioma de la interdependencia entre el cambio de la sociedad y la transformación de la comunicación pública}

Es condición necesaria para que tenga razón de ser una Teoría Social de la Comunicación, que la producción de información pública se pueda ver afectada por el cambio de las sociedades y que a su vez, a ellas les afecte. La existencia de esta interdependencia no puede ser demostrada, sino mostrada. Si se acepta la posibilidad de esa interacción como una cuestión de hecho, se constituye en el axioma inicial de la nueva Teoría.

Este libro ofrece ejemplos de esos vínculos entre modalidad de sociedad y de comunicación pública referidos a sociedades que han alcanzado niveles de desarrollo distintos. El que ahora va a utilizarse para ilustrar la existencia de tales interacciones procede de la historia mas reciente, cuando se van anudando las interdependencias entre los cambios de las sociedades burguesas y los usos de la comunicación de masas.

La evolución de estas sociedades, desde la época del capitalismo agrario y mercantil hasta la nuestra, ha ido transformando los objetos de referencia a propósito de los que en cada momento cabría comunicar. Los agentes que intervienen en el acontecer, los comunicantes que intercambian la información, los personajes mencionados en los relatos fueron variando de forma imperceptible o drástica con el devenir de las sociedades burguesas. Los medios de comunicación son y fueron gestionados de forma reglamentada por instituciones públicas o privadas en cuyo control suelen interesarse otras instituciones sociales, razón por la cual su uso mediador suele verse afectado por la dinámica política. En síntesis: la comunicación de masas, como cualquiera otra modalidad de comunicación pública, está marcada por las señas de identidad que permiten reconocer en ella a la sociedad que la utiliza. De modo equivalente en la organización y el desempeño de cada sociedad, cabe reconocer la impronta que deja el modo de producir y de distribuir la información pública.

\subsection{Estado actual de los estudios sobre la producción social de comunicación}

El reconocimiento de estas interacciones y de otras muchas más entre el estado de las formaciones sociales y las características de la comunicación pública, no plantea dificultades ni suele discutirse. El problema radica en que desconocemos todavía por qué se producen y cómo operan. Esta ignorancia tiene consecuencias teóricas y prácticas. A nivel teórico dificulta el avance de todas las disciplinas sociales que están concernidas por una explicación del cambio social y de la evolución cultural. A nivel práctico impide que las políticas encaminadas a la transformación histórica de las sociedades planteen estrategias comunicativas apoyadas en un conocimiento suficiente de lo que se está haciendo y de sus posibles efectos ${ }^{6}$.

La dinámica <Comunicación-Sociedad> aun no ha sido suficientemente esclarecida. Este estado de la cuestión obedece a varios factores:

- No existen todavía estudios seriados en los que se establezcan las correspondencias entre la evolución de las sociedades y la organización, funciones y uso de la comunicación pública. Los datos necesarios para ese trabajo aparecen desde el momento en el que la economía y el control social recurren a los medios de

\footnotetext{
${ }^{6}$ Incluso los organismos internacionales han asumido que la comunicación pública es una vía para el cambio social. Se tiene la expectativa de desarrollar la cultura, eventualmente frenar la colonización política y cultural y de impulsar el progreso de los pueblos hacia situaciones más libres y mas prosperas, transformando el uso de los medios masivos de difusión y el tratamiento de sus contenidos. Se muestra cómo son las prácticas comunicativas actuales y se las enfrenta con las necesidades de los pueblos, pero no se está muy seguro de cuáles son las alternativas correctas al actual empleo de la comunicación pública.
} 
difusión masiva, pero no han sido explotados con vistas a este nuevo uso ${ }^{7}$.

- Estos estudios de las correspondencias entre el estado de las sociedades y las modalidades de comunicación pública también son necesarios para comprender cómo se opera con la información en las sociedades de «masas». Y sin embargo las obras antecedentes son muy pocas y todas parciales razón por la cual se sigue requiriendo el esfuerzo teórico al que corresponde este libro. ${ }^{8}$

- No pueden tenerse por modelos teóricos de la producción social de comunicación tantos estudios de «comunicación social». Por lo general, los libros de «comunicación social» consisten en investigaciones puntuales o generalizaciones sobre los efectos de tal o cual medio, de tal o cual técnica de distribución de la información, de ésta o aquella estructura narrativa. En el bosque de la comunicación pública representan los árboles cortados en tantas ocasiones para un uso manipulador. Esas prácticas de la investigación sólo han dejado el calvero de una ausencia. El terreno que pretenden describir queda deformado por la acción devastadora de unos modelos teóricos instrumentales, caracterizados porque seccionan las raíces que sujetan la comunicación pública a la historia de las sociedades. La trayectoria generalmente seguida por la investigación de la «comunicación social» ha impedido comprender que la comunicación pública es ese espacio para la acción social en el que las comunidades también se han jugado, a lo largo de la historia, su viabilidad como organizaciones y por tanto su destino.

\section{EL LUGAR DE LA NUEVA TEORÍA ENTRE LAS CIENCIAS QUE ESTUDIAN LA COMUNICACIÓN}

\subsection{El Árbol de las ciencias de la comunicación}

La Teoría Social de la Comunicación que he propuesto poco tiene que ver con los estudios de «comunicación social», al menos como son interpretados por la tradición behaviorista y funcionalista. Por eso conviene hacer una delimitación de campo, que sitúe la nueva reflexión en su contexto científico.

- Entiendo que son «Ciencias de la Comunicación» todas aquellas que tienen por objeto el análisis de las interacciones en las que existe el recurso a manifestaciones

\footnotetext{
${ }^{7}$ Tal información es abundante para las primeras sociedades en las que se establece el capitalismo. Y también existe en las sociedades que tuvieron o tienen economías planificadas Incluso se puede contar con la información necesaria en sociedades dependientes donde se haya desarrollado economías de mercado, En otras sociedades del pasado y de la actualidad las fuentes resultan raras e incompletas. Ese vacío puede cubrirse en el futuro al menos en parte. Para las sociedades pre capitalistas se necesita otra lectura de los trabajos existentes en el campo de la historia política. social y económica. Para las comunidades extinguidas algunos antropólogos y etnólogos han hecho de pasada. observaciones que convendría recapitular. Para los grupos humanos cazadores, recolectores y agricultores todavía existentes, que no han terminado de ser desorganizados por las relaciones de dependencia con otros industrializados, se requerirían investigaciones para las que queda muy poco tiempo. Empeño que a pesar de la carencia de medios se está intentando sobre todo en Latinoamérica.

${ }^{8}$ Han precedido a La producción social de comunicación otros intentos teóricos que hubiesen resultado adecuados para aclarar la naturaleza de la información pública y orientar metodológicamente las investigaciones relativas a las interdependencias entre Sociedad y Comunicación, pero tuvieron otros desarrollos diferentes. Estas aportaciones suelen resultar de los diferentes encuentros entre marxismo, estructuralismo, semióticas, teoría de la información y de sistemas. Para una referencia pormenorizada puede consultarse en la bibliografía de Manuel Martín Serrano: "Métodos actuales de investigación social”. Ese esfuerzo teórico destinado a aclarar los usos de la comunicación pública estaría tanto más justificado en los países donde se ha caído en la manía del "pancomunicacionismo»: interpretación de nuestra época como la era en la que la comunicación es el motor y el contenido mismo de la interacción colectiva. Esta exageración no es inocente. Oculta una concepción acrílica y mistificadora de la acción social, poniendo en primer plano las modalidades tecnológicamente mediadas de relación social y relegando al fondo sus contenidos y su naturaleza.
} 
expresivas ${ }^{9}$.

- La «Teoría de la Comunicación» tiene que proporcionar el paradigma que sirva de marco al conjunto de las Ciencias de la Comunicación. Le corresponde ocuparse de las características que diferencian a la interacción expresiva de otras modalidades de interacción que recurren a actos ejecutivos. El nivel de análisis tiene que tomar en cuenta toda posible manifestación de las relaciones comunicativas; tanto si se producen entre Actores animales como humanos; y con independencia de que la información esté biológica o tecnológicamente expresada ${ }^{10}$.

- Las Ciencias de la Comunicación penetran en el ámbito de las Ciencias Humanas cuando seleccionan como objeto de estudio las comunicaciones entre Actores que son hombres. Una «Teoría de la Comunicación Humana» justifica su especificidad porque nosotros no comunicamos sólo a propósito de lo que los animales comunican, ni sólo como ellos lo hacen. La comunicación humana incluye entre sus objetos de referencia además todo el ecosistema material, social, gnoseológico y axiológico producido por el propio ser humano. Incorpora al sistema de interacción productos que son fabricados ex profeso para cumplir una función expresiva (por ejemplo una carta) y se sirve de herramientas que amplifican el alcance en el espacio de las señales (por ejemplo el teléfono) y prolongan la duración en el tiempo de las manifestaciones comunicativas (por ejemplo los magnetoscopios).

- Cuando la comunicación humana se sirve de tecnologías que permiten multiplicar los productos comunicativos (como ejemplos la imprenta, Internet): implica a numerosos sujetos o se refiere al acontecer que concierne a la comunidad, el estudio de estas prácticas le reserva un lugar a las Ciencias de la Comunicación entre las Sociales.

Es posible aclarar cuál es el modo de preguntar que genera ese otro punto de vista que otorga su pertinencia a esta nueva disciplina:

La Teoría Social de la Comunicación, como toda ciencia, va en busca de leyes generales, categorías universales y modelos predictivos. Por haber ligado el estudio de la sociedad y el de la comunicación, se pregunta por el cambio, o para ser más precisos por el intercambio entre dos cambios: el que se produce en las formaciones sociales y el que se manifiesta en las modalidades de comunicación pública. Siempre toma en consideración la

\footnotetext{
${ }^{9}$ Las manifestaciones expresivas en las interacciones que no están tecnológicamente mediadas recurren a las capacidades expresivas del cuerpo, tales como la voz o la gestualidad, Y cuando se utilizan medios tecnológicos, a instrumentos que amplifican o traducen las señales como el teléfono y el ordenador. Las manifestaciones expresivas se reconocen porque indican un estado, una necesidad, un objeto, etc. En cualquier caso la indicación comunicativa supone que en la interacción se realicen «actos expresivos» aquellos comportamientos de los Actores en los que sus energías están orientadas a producir información destinada a otros Actores. A tenor de lo dicho los actos expresivos se distinguen de los «actos ejecutivos» porque el trabajo del Actor aplica su energía a la obtención de expresiones y no directamente a modificar ni a la entidad designada ni al Otro u Otros Actores. El estudio detallado de este tema se encuentra en “Teoría de la Comunicación. Epistemología y análisis de la referencia”. Manuel Martín Serrano et Alt.

${ }^{10}$ La Teoría de la Comunicación que proporciona la referencia epistemológica de esta obra: $<$ La Teoría Social de la Comunicación> no es < La Teoría de la Comunicación>, porque carece del alcance epistemológico que define a esta última. Muy al contrario la posibilidad de la primera, presupone un desarrollo suficiente de la segunda. Esa es la razón por la que he indicado que un libro de las características que éste tiene ha requerido previamente otra reflexión más general. Hay que apoyar el estudio de las funciones y los usos de la Comunicación Institucional en un marco epistemológico que explique cuál es la naturaleza específica de la comunicación, su ámbito, funcionamiento y componentes. Aquí no se da cuenta de ese trabajo de base porque está publicado en otros libros*. No obstante en este texto se han introducido las aclaraciones teóricas necesarias cada vez que se da paso a una interpretación general de los fenómenos comunicativos que se manifiestan a nivel de las sociedades.

* La primera formulación sistemática que el Autor ha hecho de una Teoría de la Comunicación que sea el soporte epistemológico del conjunto de las ciencias que estudian interacciones comunicativas está recogida en "Teoría de la comunicación. Epistemología y análisis de la referencia”.
} 
producción de comunicación como una actividad histórica, porque las sociedades que las producen también lo son y porque la validación de sus hipótesis se realiza comprobando si el curso que siguen los Sistemas de Comunicación en el desarrollo de las comunidades se ajusta a las previsiones de la Teoría.

\subsection{La denominación para los estudios referidos a la producción social de comunicación}

El rotulo al que se suele recurrir para referirse a todos los trabajos que tengan alguna dimensión colectiva, es «comunicación social». Pero tal denominación me parece confusa e inapropiada para distinguir a los temas que tienen que ver con la producción social de comunicación. Convendría otro título con el que se pudiese percibir la apertura que tienen las Ciencias de la Comunicación a objetos de investigación que comparten con otras Ciencias Sociales. Los reparos que pongo se justifican por las siguientes razones:

a) «Comunicación social» dice demasiado si se fundamenta en el uso «no privado» de la información. Toda comunicación humana, incluida la privada, es social en razón de la naturaleza de los Actores. Las respectivas pertinencias de los estudios de «Comunicación Humana» y de «la Comunicación Institucional» quedan difuminadas.

b) «Comunicación social» dice demasiado poco si se refiere sólo a las interacciones que están tecnológicamente mediadas, es decir aquellas en las que se utilizan «los medios». La comunicación pública existe y existió como forma de interacción social sin necesidad de herramienta mediadora alguna. En muchas comunidades funcionan Sistemas Institucionales de comunicación con el mero uso de la palabra hablada ${ }^{11}$.

c) «Comunicación social» delimita un campo de estudios por el objeto material. Esa sola razón muestra que es una identificación científicamente incorrecta.

d) Finalmente «comunicación social» es una denominación históricamente asociada a una concepción propagandística, cuando no desembozadamente manipuladora del recurso a la comunicación pública. Enfoque más bien asocial con el que en la actualidad se designan mayormente, los usos comerciales, políticos y militares de los medios de difusión, cada vez más contaminados por materiales comunicativos embrutecedores, amenazantes o mistificadores. Conviene colocar en su lugar los saberes instrumentales que han desarrollado tales técnicas para el engaño y la opresión y tratar de que no sigan suplantando al conocimiento científico.

La existencia de información que se produce, distribuye y usa de forma institucionalizada y que concierne a los aconteceres que interesan a la comunidad en su conjunto, es la razón por la que las Ciencias de la Comunicación pueden tener una disciplina que pertenece al ámbito de los estudios sociales. Las consideraciones que he hecho explican que cuando propongo que se denomine «Teoría Social de la Comunicación» al estudio de esas prácticas comunicativas, esa inversión semántica no la hago por un prurito de originalidad. Tomo en cuenta razones heurísticas e históricas, a las que cabe añadir el respeto a la coherencia terminológica. En la fórmula que sugiero, «social» delimita a la vez el enfoque teórico y el campo de investigación, tal como debe de hacerse para definir una disciplina.

\footnotetext{
${ }^{11}$ Los Sistemas de Comunicación Pública en los que todavía no existe una infraestructura tecnológica se describen en la página 74 y sigs. de este mismo libro.
} 


\section{FUNDAMENTOS EPISTEMOLÓGICOS Y PARADIGMÁTICOS DE LA TEORÍA SOCIAL DE LA COMUNICACIÓN}

\subsection{El paradigma científico que corresponde al enfoque de esta obra}

Toda obra teórica presupone un modelo general que el autor tiene por el punto de vista adecuado para enfrentarse con el objeto de estudio. Son paradigmas utilizados en las Ciencias Sociales, entre otros, el Behaviorista, el Estructuralista, el Dialéctico. El que yo tengo por pertinente lo he denominado «de la Mediación». La mediación pretende ofrecer un paradigma adecuado para estudiar todas aquellas prácticas, sean o no comunicativas, en las que la conciencia, las conductas y los bienes entran en procesos de interdependencia. El investigador no puede recurrir en estos casos a modelos meramente cognitivos, exclusivamente de comportamiento, o solamente de producción. La necesidad de un enfoque basado en el análisis de la mediación se hace sentir cuando el manejo de la información, de los actos, de las materias, se manifiesta como una actividad que no puede ser disociada ni analizada por partes. La producción de información destinada a la comunicación pública es una de esas actividades ${ }^{12}$.

\subsection{El recurso a las fuentes. Las Ciencias Sociales que anticipan los estudios de la producción social de comunicación}

Ninguna teoría nace en un vacío de ideas. Aunque la interdependencia entre las sociedades y la comunicación pública sea un objeto de estudio que hasta ahora no se había independizado como una ciencia específica, esta nueva reflexión puede madurar en la mejor solera. Los antecedentes teóricos más pertinentes se encuentran en los trabajos que se ocupan de las relaciones entre cultura y sociedad, uno de los temas que dan nacimiento a las Ciencias Sociales. Todas las escuelas importantes -Marxista, Organicista, Funcionalista, Estructuralista y Sistemática, entre otras- han propuesto alguna interpretación de esas relaciones. En consecuencia existe un capital teórico que se debe utilizar en la fundación de una Ciencia Social de la Comunicación digna de ese título, aunque las categorías y los modelos procedentes de la tradición sociológica tengan un carácter más general y los diferentes análisis discrepen notablemente.

El recurso de las fuentes es una labor necesaria, pero insuficiente, para que la Teoría Social de la Comunicación adquiera un estatuto científico propio en el marco de las Ciencias que estudian fenómenos sociales. El estudio de los intercambios <sociedad-comunicación> requiere de la elaboración de modelos teóricos específicos, que sean adecuados para diseñar investigaciones paradigmáticas, es decir destinadas a la validación de las hipótesis contenidas en la nueva Teoría ${ }^{13}$.

\footnotetext{
12 El paradigma de la mediación es un modelo que trabaja con los intercambios entre entidades materiales, inmateriales y accionales. Esta especificidad explica que recurra a análisis lógicos, y que cuando se aplica a procesos históricos, se apoye en una lógica dialéctica y genere modelos dialécticos. Las características del análisis de la mediación se van a manifestar en este libro como en otros que le han antecedido. El lector interesado en conocer el origen del concepto y de los modelos de la mediación puede leer la obra en la que se ha desarrollado ese paradigma (cfr. de Manuel Martín Serrano:” La mediación social”) . No obstante en cuanto sea necesario para comprender la aplicación que del citado paradigma se hace en este libro, se ofrecerán las explicaciones necesarias a lo largo de la exposición.

${ }^{13}$ Estos trabajos de base todavía no se han realizado en el dominio de la comunicación pública. A diferencia de lo que sucede, por ejemplo, en Antropología Cultural, en Sicología Cognitiva o en Economía Política, en el estudio de los procesos y de los sistemas comunicativos que existen en las sociedades, todavía la teoría suele ir por una parte y las investigaciones por otra. Nuevamente se podría relacionar una lista larga de títulos que se ocupan de las funciones y de los efectos de la comunicación de masas tanto en las sociedades dominantes como en las dependientes. En el conjunto de estas obras se reconocen los enfoques alternativos que distinguen a los Evolucionistas, Funcionalistas, Estructuralistas o Marxistas. Pero estas diferencias generalmente resultan de las diversas posturas axiológicas de los autores, en vez de distinguir paradigmas lógicos diferentes.
} 
La mayor parte de los temas que interesan a la Teoría Social de la Comunicación han sido mencionados y en ocasiones discutidos en otros contextos científicos. Lo mismo sucedió antes de que cada ciencia social se hiciese independiente del tronco que a todas las sostiene. No obstante sigue abierto el estudio sistemático de todas las cuestiones cruciales para comprender las diferentes relaciones de interdependencia que existen, entre el cambio social y la transformación de la comunicación pública ${ }^{14}$. En todo caso la existencia de un espacio científico que le es propio a la Teoría Social de la Comunicación no dispensa de la necesidad de informarse en diversas fuentes ${ }^{15}$.

\section{LOS TEMAS}

El catálogo de las cuestiones que conciernen a la Teoría Social de la Comunicación es de naturaleza abierta y por tanto incompleta y provisional. A medida que se va ampliando el conocimiento del objeto aparecen nuevos campos de reflexión.

Existe un repertorio de temas que cabria denominar fundacionales, porque al esclarecerlos se está construyendo la armazón de la disciplina. Son los siguientes:

- ¿ ¿En qué se diferencia, y cómo llega a diferenciarse, la comunicación pública de las otras modalidades de comunicación social?

- ¿Cuántos tipos diferentes de Sistemas Comunicativos han existido y existen para gestionar la comunicación pública? ¿Qué características los diferencian?

- ¿Cómo se integran institucional y funcionalmente la producción y reproducción social con la producción y reproducción de comunicación pública a lo largo de la historia?

- ¿Qué niveles del Sistema Social pueden afectar a la organización y al funcionamiento del Sistema de Comunicación y viceversa?

- La naturaleza de las interacciones entre ambos Sistemas y sus efectos, ¿se ajustan a constricciones o principios que tengan un valor histórico permanente y una aplicación universal en todas las sociedades?. ¿Si existen, cuáles son tales principios?

- La aparición, transformación y desaparición de los Sistemas de Comunicación Pública ¿obedecen a leyes históricas? ¿Cuáles son esas leyes?

- ¿ ¿En qué momento y con qué efectos comunicativos y sociales la comunicación pública comienza a servirse de productos comunicativos fabricados ex profeso para ser soportes de información?

- ¿QQué características diferencian la producción de comunicación y los propios productos comunicativos de otras producciones y de otros bienes?

\footnotetext{
${ }^{14}$ Por ser tan joven la investigación en este campo ha alcanzado la altura de una hermenéutica de los fenómenos comunicativos, lo cual no ha sido un logro pequeño; pero todavía previo al nivel que requiere una Ciencia Social de la Comunicación. Por eso un comunicólogo Funcionalista todavía no es alguien que, como el antropólogo Funcionalista, haya encontrado en la sociedad de masas a su comunidad Trobiant, ejemplo con el que justificar sus hipótesis del ajuste sociocultural; ni un comunicólogo Marxista alguien que, como el historiador de su misma escuela, haya encontrado ya en la comunicación pública ese sistema de formación y de cambio de valores con el que apoyar sus postulados materialistas-dialécticos.

${ }^{15}$ No cabe dejar fuera de esa atención a los fenómenos de la comunicación pública a las ciencias psicológicas (por ejemplo la Psicología Cognitiva), antropológicas (por ejemplo la Antropología Cultural), sociológicas (por ejemplo la Teoría del Cambio Social), lógicas (por ejemplo la Semiótica y la Teoría de la Información), comunicativas (por ejemplo la Semiología o la Investigación de la Comunicación de Masas). Pero ninguna de ellas podía abarcar todos los temas por razón de su particular especificidad. Tampoco pretendían generar una teoría que articulase todos los usos sociales de la comunicación pública en un mismo corpus científico, por razón de que sus respectivas referencias epistemológicas son otras.
} 
- ¿Cómo se articula en el producto comunicativo su doble naturaleza de cosa fabricada y de representación narrativa de lo que acontece?

- ¿Cuáles son las categorías, los métodos y las técnicas necesarias para investigar la naturaleza y las funciones de los productos comunicativos, tanto en su dimensión de cosas materiales como en su dimensión de narraciones?

- Los productos comunicativos ¿utilizan un repertorio amplio o reducido de modelos de estructuras narrativas para dar cuenta de la información sobre el acontecer? ¿Cuáles son y cómo son esos modelos?

- Los modelos de estructuras narrativas utilizados en la comunicación pública ¿son universales o particulares? ¿Por qué tienen unos componentes y una configuración determinada en vez de otras alternativas? ¿Cuáles son los cambios del entorno, del Sistema Social, de las tecnologías que transforman esos modelos o que los perpetúan?

- La transformación de la visión del mundo que se ofrece en la comunicación pública, ¿se anticipa, acompaña o sigue a la transformación de la sociedad? ¿Qué sucede cuando las sociedades se dotan de instituciones mediadoras expresamente ocupadas en el control de las representaciones del mundo a través de la comunicación pública?

- ¿Existe efectivamente la posibilidad comunicativa de cambiar las visiones del mundo en la sociedad, sin la previa revolución de las estructuras narrativas?. ¿Qué modificaciones de los modelos utilizados en los relatos pueden tenerse por transformadoras de la concepción del mundo que se ofrece en la comunicación pública?. Pero esa creación de estructuras narrativas nuevas para los relatos que adquieren el status de representaciones colectivas, ¿Puede llevarse en la práctica sin el previo o el simultáneo cambio de la Formación Social, a nivel político y económico?

- ¿Cómo opera, si es que opera, el proceso que relaciona la transformación del orden social y el cambio de las visiones de la realidad?. Supuesta esa interdependencia, ¿responde a afectaciones de naturaleza generativa, funcional o dialéctica?

Estos temas y los que ellos generan son los que se abordan en este libro. El tratamiento de algunos todavía no es completo, o bien porque aún no existen los estudios históricos previos que permitan conclusiones definitivas, o bien porque piden la reiteración de investigaciones específicas que requieren más tiempo, En cualquier caso he intentado que el sistema teórico ofrezca un lugar para ubicar a todas las cuestiones enunciadas.

\section{ELECCIÓN DEL OBJETO PARADIGMÁTICO DE INVESTIGACIÓN}

Las investigaciones diseñadas para probar la validez de las propuestas teóricas tienen que ser estudios paradigmáticos ${ }^{16}$.

¿Cuáles serían las sociedades objeto de investigación cuya evolución, a nivel de su formación sociopolítica y de sus modalidades de comunicación pública, conviene elegir como banco de pruebas para una Teoría Social de la Comunicación?. Me pareció que la elección adecuada correspondía a las sociedades surgidas de las revoluciones burguesas. Esta decisión puede justificarse por ser lo más viable. Ciertamente es ventajoso para toda ciencia que empieza trabajar con el ejemplo en el que hay más posibilidades de disponer de datos. Pero además estas sociedades cumplen dos requisitos que convienen para una investigación paradigmática y que

\footnotetext{
${ }^{16}$ Se dice en lenguaje mas técnico que son obligatorios los diseños experimentales o cuasi-experimentales para establecer de qué modo se van a falsificar las afirmaciones de las teorías. En los diseños dialécticos la comprobación de la verdad o falsedad de los postulados, leyes, conclusiones ,se puede llevar a cabo con el paso del tiempo, cuando se vayan cumpliendo los cambios históricos que se hubiesen enunciado en la teoría.
} 
no satisface ninguna otra:

$1^{\circ}$. Ha sido en las formaciones sociales que son hijas de las revoluciones burguesas en donde las interacciones entre la organización social y la comunicación pública han adquirido el mayor grado de desarrollo y de complejidad. El estudio de los casos históricos más evolucionados sirve en cierta medida, para comprender los que le antecedieron o se desenvolvieron por otras líneas de interdependencia distintas.

$2^{\circ}$. Las sociedades capitalistas son todavía comunidades en marcha, lo mismo que las modalidades de comunicación pública que en ellas se han institucionalizado. Han pasado por etapas distintas bien definidas, referidas a los períodos agrarios-mercantiles, preindustriales, industriales y ahora la mayoría de ellas están enredadas en la globalización económica que controlan los monopolios. La Teoría que tenga utilidad social debe de anticipar cambios históricos a partir de sus leyes, que si son ciertas y correctas tendrán su confirmación precisamente cuando el cambio anunciado y todavía no producido llegue a cumplirse.

\section{LA INVESTIGACIÓN QUE SE UTILIZA PARA FALSIFICAR LA TEORÍA}

La oportunidad de llevar a cabo una investigación paradigmática en una sociedad capitalista que estaba en un momento de cambio se presentó en España cuando el Régimen Franquista, totalitario y autocrático, fue sustituido por la Monarquía, parlamentaria y democrática ${ }^{17}$.

\subsection{Diseño de la investigación}

Se utilizaron para probar la teoría materiales comunicativos aparecidos en la Televisión y en la Prensa tanto durante los últimos años del Franquismo como durante los primeros años de la Monarquía Parlamentaria. ${ }^{18}$. Desde el punto de vista metodológico se requiere disponer de la producción de un medio de comunicación para el análisis experimental y de la de otro medio diferente para el control de los análisis. El estudio comparado de la producción comunicativa de la Prensa y de la Televisión permite ese diseño, porque cada medio tiene características expresivas diferentes. ${ }^{19}$

Con los datos obtenidos se realizaron las siguientes comparaciones:

\footnotetext{
${ }^{17}$ El uso de la comunicación pública se hace más transparente cuando puede estudiarse en una sociedad donde se están desarrollando transformaciones histéricas y con ellas reajustes entre la comunicación pública y el nuevo modelo de sociedad. España cuando pasó del Franquismo a la Monarquía Parlamentaria fue un adecuado banco de pruebas porque disponía de instituciones y de tecnologías comunicativas tan modernas como los demás países industrializados, pero en cambio padecía un régimen autoritario que ponía trabas pre liberales a su uso. Por eso aunque la transición española de la dictadura a la democracia sea una peripecia política irrepetible y represente un corte muy limitado en el espacio y en el tiempo, el estudio de ese tránsito posee un valor estratégico para analizar las relaciones entre Sistema Social (Capitalista) y modo de producción de comunicación pública (de Masas). Los detalles metodológicos y los resultados de esta investigación se encuentran en la edición primera de este mismo libro.

${ }^{18}$ La investigación de referencia fue "Comparación entre los contenidos de la información política en el franquismo y en la monarquía parlamentaria”, Se analizaron en la Prensa y la TV 1.500 unidades (temas) para identificar la representación del mundo en cada etapa política. Los diseños para este trabajo derivan de la experiencia obtenida con otras investigaciones precedentes entre ellas "Análisis del contenido de los programas de TV española”, "Análisis de la Visión del Mundo en la TV”, "L 'Ordre du monde a travers la TV”, "La imagen de los líderes políticos”. El diseño, los modelos y las técnicas se fueron gestando en precedentes trabajos, donde se desarrollan propuestas metodológicas para el estudio de la mediación. Entre ellos "Nuevos métodos para estudiar la estructura y la dinámica de la enculturización”, “Un método lógico para analizar el significado”, “Métodos de análisis de contenido". La referencia detallada aparece en la bibliografía de obras del Autor.

${ }^{19}$ La Prensa es un medio abstracto y acrónico; la Televisión un medio que puede ser icónico y sincrónico. En este mismo libro se aclara el significado de estos conceptos. Al contar con ambas fuentes de información se evita el riesgo de caer en una falsa inferencia, que se produciría si se atribuyeran a influencias sociales comportamientos comunicativos de los medios que en realidad obedecerían a peculiaridades expresivas.
} 
a) Entre etapas políticas distintas (diferencias y semejanzas entre la producción comunicativa del período Franquista y del período Monárquico-Parlamentario).

b) Entre medios de comunicación pública distintos (diferencias y semejanzas entre la producción comunicativa de la Televisión y de la Prensa, en cada etapa política).

c) Entre ámbitos de referencia diversos (diferencias y semejanzas entre la producción comunicativa referida a temas sociopolíticos y la relativa a otros temas tales como la familia, el consumo, etc.; tomando en cuenta el medio en el que difunden y la etapa política durante la que se producen).

Este diseño se corresponde con el modelo canónico de análisis que está descrito en el capítulo seis de este libro.

\subsection{Las técnicas de investigación}

La Teoría Social de la Comunicación se ocupa de un objeto de estudio cuya naturaleza es histórica: la producción social de comunicación. Las metodologías que se adecuan a un objeto de estas características tienen que ser aptas para captar las independencias, las correspondencias y las contradicciones que puedan observarse entre el cambio de las sociedades y la transformación de la comunicación pública. Esos requisitos los cumple la metodología dialéctica ${ }^{20}$.

Precisamente por estar trabajando con un objeto dialéctico, las técnicas de análisis que se utilicen deben de recurrir a todo sistema de recuento y discriminación que permita captar qué cambios de grado y qué cambios cualitativos aparecen en la producción de comunicación pública y en el uso de los media con el paso de una etapa política a otra. Esas técnicas proceden en unos casos de la estadística, en otros de la lógica, en ocasiones de la teoría de sistemas ${ }^{21}$. A veces las técnicas que se utilizan han sido desarrolladas ex profeso por quien escribe para resolver las necesidades específicas de una investigación que trabaja con productos comunicativos ${ }^{22}$. En los demás casos, técnicas similares han sido utilizadas antes por Behavioristas, Funcionalistas, Sistémicos y Estructuralistas en el marco de sus respectivos planteamientos.

Las técnicas de investigación son una parte constitutiva del objeto de análisis. Por eso es cierto que pueden arrastrar tras de ellas a la teoría e incluso ocupar su lugar, si acaso la investigación carece de un paradigma al que se remita la interpretación de los datos. Todo especialista en metodología de las ciencias sociales sabe que las técnicas no son inocentes. Yo mismo he descrito con detalle sus perversidades cuando me he ocupado de ese tema ${ }^{23}$. Pero coincido con los fenomenólogos en que una investigación crítica, para serlo, antes que nada tiene que ser capaz de describir el objeto tal cual se muestra.

\footnotetext{
${ }^{20}$ Cuando se hace alguna aplicación en este libro del método dialéctico se ofrecen las oportunas descripciones y explicaciones. La primera edición incluye anexos específicos referidos a los diseños y las metodologías. Hay desarrollos sistemáticos de estos métodos y de su paradigma en otras obras del Autor: Cf. "Epistemología de la dialéctica social”, "La mediación social”, "Métodos actuales de investigación social”, “Trabajos a propósito de otros métodos de investigación en Ciencias Sociales”,”Economía política y comunicación pública”.

${ }^{21}$ Estos programas tienen dos ventajas: la primera es que permiten manejar un volumen de datos tan grande como el que aquí se utiliza, facilitando la distinción entre la información que es relevante y la que carece de interés; y la segunda ventaja consiste en que están diseñados para operar con sistemas de variables, facilitando el conocimiento de las relaciones que existen entre estructuras de datos y no sólo entre datos independientes. Los textos del Autor dónde se describen estas metodologías aparecen referenciados en la Bibliografía y son los siguientes: "Propuesta de un modelo del espacio y la relación para investigar el cambio social, y aplicación al análisis sociológico del complejo de Edipo", "Nuevos métodos para estudiar la estructura y la dinámica de la enculturización”, "La mediación social”, "Un método lógico para analizar el significado", “Trabajos a propósito de otros métodos de investigación en Ciencias Sociales", "Un modelo metodológico para investigar los efectos socioculturales de los media".

${ }^{22}$ El lector que esté interesado en las técnicas de análisis lógico que ha diseñado el Autor para la disección de los productos comunicativos, las puede encontrar en la primera edición de esta misma obra.

${ }^{23}$ Cfr. Manuel Martín Serrano: "Métodos actuales de investigación social”.
} 
Un ejemplo procedente de las investigaciones realizadas para escribir este libro ayuda a aclarar esta idea. La unidad que he utilizado para descomponer los relatos de la Prensa y de la Televisión es el rol. El rol es una venerable categoría que procede de la sociología de Comte y que a través del funcionalismo de Durkheim ha pasado a ser elemento muy definitorio de la sociología estructural-funcional norteamericana ${ }^{24}$. Cuando se reduce la acción de los sujetos sociales al desempeño de sus roles, se tiene una concepción cosificada e instrumentada de los comportamientos y de las prácticas sociales. Suele designarse a esa clase de reduccionismo con el nombre de reificación ${ }^{25}$. Pero sucede que tal reificación es la que precisamente existe en las narraciones de los medios de comunicación de masas, donde los personajes se resuelven en un ramillete de roles ${ }^{26}$. Por lo tanto si se pretende -y aquí se pretende- sacar a la luz una práctica narrativa mistificadora que se basa en la rolificación, es obligado que los objetos de análisis sean los roles y no solo los sujetos sociales reales que en ellos se disuelven y desnaturalizan. La interpretación de esos análisis será funcionalista, estructuralista, sistémica o dialéctica según como luego el autor ponga en relación esos datos, procedentes del modelo real que se encuentra en los productos comunicativos, con la sociedad que los produce y con el uso que en ella se haga de la comunicación pública ${ }^{27}$.

\section{CARACTERÍSTICAS DE ESTA EDICIÓN DE «LA PRODUCCIÓN SOCIAL DE COMUNICACIÓN»}

La presente redacción de este libro es la tercera y también la más reducida. Para preparar el nuevo texto he considerado que será la última revisión que llevaré a cabo. A partir de este supuesto he realizado dos tareas. Una de ellas, para mejorar la forma de exposición con el objeto de que el texto se adecue a las actuales funciones que cumple. Otra, para actualizar y desarrollar los contenidos, tomando en cuenta los cambios sociales que se han producido y las transformaciones de los usos de la comunicación, Para ello he tomado en cuenta las aportaciones de posteriores estudios de la comunicación pública. Principalmente proceden de investigaciones que he seguido realizando desde que escribí la primera edición de esta obra hasta la fecha, todas ellas diseñadas a partir de La producción social de comunicación. ${ }^{28} \mathrm{Me}$

\footnotetext{
${ }^{24}$ La evolución del concepto y el uso de la categoría rol pueden consultarse, en la bibliografía de Manuel Martín Serrano, "Comte, el Padre negado".

${ }^{25}$ Sobre este tema hay un análisis detallado en "Modelo del espacio y de la relación para investigar el cambio social”. Cf. en la bibliografía de Manuel Martín Serrano.

${ }^{26}$ El análisis de la rolificación está publicado en las precedentes ediciones de este mismo libro dentro de los capítulos referidos a "los roles que se les encomiendan a los actores".

${ }^{27}$ Queda lejana aquella etapa de ignorancia teórica, en la que cierta izquierda se entretenía en dividir las técnicas en «reaccionarias» y «revolucionarias», cayendo del primer lado varias de las que había utilizado el mismo Carlos Marx, como podrían haber comprobado si dichos dogmáticos se hubiesen animado a leer El Capital. Esta tontería llevó a discusiones cómicas como aquella de si la estadística era o no contrarrevolucionaria. No obstante en algunos países de nuestra lengua, a tenor de lo que se publica, parece que el tema del uso de las técnicas que convienen a una investigación crítica y no manipuladora de los fenómenos comunicativos todavía no está resuelto. Por ello parece oportuno hacer algunas observaciones generales al respecto, aunque en esta edición no se haya incluido el detalle de las técnicas de investigación. Por eso creo que la pregunta que en una conferencia ante colegas de Latinoamérica se me formuló, de cómo una Teoría Social de la Comunicación que se apoyaba en un paradigma dialéctico (el de la Mediación) recurría a técnicas de análisis también utilizadas por la sociología académica, podría invertirse y servir como respuesta: ¿cómo le será posible a cualquier teoría dialéctica excluir de su investigación las categorías y las escalas que conforman y reifican el objeto que está investigando? No son las técnicas de investigación las que se dividen en «dialécticas» $\mathrm{y}$ «no dialécticas», sino los paradigmas.

${ }^{28}$ La producción social de comunicación es un cierre para una etapa de producción teórica y metodológica. Y por eso mismo abrió un nuevo horizonte a la investigación. Se han sumado a esta propuesta reconocidos investigadores en España, Latinoamérica, EE.UU. y varios países de Europa, sobre todo en Francia y Alemania. Manuel Martín Serrano ha continuado investigando sin interrupción por si mismo y con sus colaboradores los cambios en la comunicación pública. De estos trabajos están referenciados en la bibliografía del Autor los siguientes: "Funciones de la comunicación personal, institucional y de los MCM en los jóvenes", "Prospectiva del uso de los MCM", "La participación de los medios audiovisuales en la construcción de la visión del mundo de los niños", "La
} 
refiero brevemente a la naturaleza de cada una de esas revisiones.

\subsection{Revisión didáctica}

He tomado en cuenta el uso que se está haciendo de la obra como un texto de estudio por profesores y estudiantes de las escuelas y facultades de comunicación en España y en Latinoamérica. Sigue siendo un libro completo, pero diseñado para facilitar la tarea de aquellos lectores o lectoras que estén interesados en los conceptos, en los métodos y en las propuestas de la Teoría Social de la Comunicación y no tanto en la forma en la que se validó esa teoría y se probó esa metodología. En consecuencia he sustituido siempre que resultaba posible un término técnico por otro más usual. Y para aligerar la lectura he llevado a notas la mayoría de los párrafos que amplían cuestiones teóricas o metodológicas.

Las mismas razones me han animado a reducir cuanto me ha sido posible el tamaño del libro. Esta edición se centra en aquellas partes de la obra que resultan necesarias para exponer los contenidos teóricos y metodológicos. Los capítulos excluidos corresponden a las aplicaciones y a los estudios empíricos. El lector o la lectora interesados pueden consultar esos materiales en las ediciones precedentes. ${ }^{29}$

La producción social de comunicación es un texto cuya validez depende de la medida en la que los cambios históricos demuestren la utilidad de sus conceptos, la pertinencia de sus métodos y el acierto de sus predicciones. Esa circunstancia justifica que haga ahora una sucinta referencia a los orígenes y a la naturaleza de esta obra, porque han determinado el tratamiento de esta tercera redacción.

\subsection{Revisión histórica}

La producción social de comunicación desarrolla unas tesis relativas a las afectaciones que se observan entre los cambios sociales y las transformaciones de las representaciones colectivas. Esas tesis están publicadas a partir de 1972 en varios artículos de revistas especializadas y alcanzaron la mayor difusión en las sucesivas ediciones de "La Mediación Social ${ }^{30}$.

Decía en aquellos escritos que se estaba gestando una forma históricamente nueva de producción y de reproducción social. Que la información, la acción social y la organización, iban estableciendo vínculos funcionales cada vez más fuertes entre ellas, de forma que tal

transformación en el uso de la comunicación social por los españoles” (serie de investigaciones durante la década de 1990 al 2000, que continúa), "El cambio social y la transformación de la comunicación”, ”Los cambios acontecidos en las funciones de la comunicación y en el valor de la información”, "La comunicación pública y la supervivencia”, "La imagen de los varones y de las mujeres en la TV”, "Las demandas comunicativas de los jóvenes”, "La sociedad lectora”, "Contenidos y funciones de los Reality Show”, "Nosotras y vosotros, según nos ve la televisión”, "Los valores de los jóvenes y el universo axiológico de la televisión”, "Producción de la Información Pública en Televisión”, "Valoración de la información pública de la televisión en las audiencias”, "Las transformaciones sociales vinculadas a la era audiovisual”, "Lecturas y lectores entre la juventud española”.

${ }^{29}$ La primera edición de esta obra ha aparecido en Madrid, en 1986, publicada por la misma editorial en su colección «Alianza Textos» con el número 102. Incluye la investigación relativa a la transformación de la comunicación pública con el paso de Franquismo a la Monarquía Parlamentaria, que se utilizó para probar y validar los planteamientos teóricos y las conclusiones. El investigador profesional de las ciencias sociales encontrará en la primera edición de este libro el desarrollo detallado de los diseños, variables, categorías, tratamiento de los datos, controles de representatividad, significación y validez, criterios de inferencia y de falsificación que puede necesitar para planear sus propias investigaciones. La segunda edición se editó simultáneamente en España y en México en 1994 y cuenta con varias reimpresiones. Fue revisada para presentar de un modo más completo y sistemático la descripción de los métodos de investigación. En esta tercera edición se ha eliminado la parte del libro titulada "la investigación de la comunicación publica a partir de los productos comunicativos". En ella se contienen las aplicaciones y los resultados de la investigación que sirvió para probar el modelo.

${ }^{30}$ En La Mediación Social el Autor propone el paradigma de la mediación, al que hace referencia en el epígrafe 4.1 de esta introducción, como el soporte teórico y metodológico de La producción social de comunicación. 
interdependencia terminaría minimizando la autonomía de cada uno de esos subsistemas sociales. Y que las ciencias especializadas en cualquiera de esos tres componentes se verían en la necesidad ineludible de ocuparse al tiempo del funcionamiento de los otros dos. Para lo cual se requerían nuevos paradigmas más intersistematicos y nuevos métodos más comprehensivos, tales como los que se proponían en "La mediación social”.

El modelo de la mediación se gestó en un ambiente intelectual y político que esperaba la aparición de grandes cambios históricos y que se preparaba con optimismo para la transformación de las formas de vida, de las relaciones personales e institucionales, de los valores y de las metas colectivas. ${ }^{31}$

Esas tesis requerían de una teoría que las articulase, de una metodología que las instrumentase y de unas investigaciones que las probasen. En eso consistía precisamente $L a$ producción social de comunicación. La primera versión del libro la escribí entre 1976 y 1986. Una apoca comparativamente mucho menos creativa que la década precedente en el campo de la teoría social, tal vez porque se vislumbraba en el horizonte un ciclo histórico de cambios tecnológicos que no se sabía muy bien en qué iban a desembocar. Se tenía la certeza de que en su mayor parte tales transformaciones tendrían que ver con la producción, la transmisión y el uso de la información. Sólo algunos autores y entre todos Abraham Moles con más autoridad que ninguno, nos aventurábamos a imaginar cuales serian los escenarios para las interacciones sociales, cuando la banda ancha permitiese la integración de las tecnologías informáticas y comunicativas en un único sistema y las correspondientes tecnologías se hubiesen incorporado a los hogares. Pero aun resultaban inimaginables los usos cotidianos que desde albores del siglo XXI hacemos de la red de redes para las comunicaciones privadas e institucionales ${ }^{32}$

El tiempo transcurrido entre la primera redacción de este libro y ésta tercera es corto. Pero la naturaleza y la intensidad de los cambios acaecidos en la comunicación pública son muy relevantes. Por esa razón comienzan a ser verificables los modelos, las leyes y las interpretaciones que están propuestas en La producción social de comunicación. A partir de ahora es posible comprobar la capacidad de predicción que tiene la Teoría Social de la Comunicación cotejando el testo original con el actual contexto social y comunicativo ${ }^{33}$. Para que esa verificación revele los aciertos y los errores, la completitud de esta obra y sus insuficiencias, he mantenido en su integridad todos los contenidos que deben de ser objeto de validación respetando las redacciones y los gráficos originales. Concretamente he reproducido en su integridad y tal como aparecieron en las ediciones precedentes, los planteamientos, diseños, enunciados, deducciones y conclusiones.

Una vez satisfecho este objetivo he pretendido que esta tercera redacción de " $L a$ producción social de comunicación” aclare mejor y desarrolle con más profundidad la Teoría Social de la Comunicación. La relectura del texto imponía algunos cambios y animaba a otros:

\footnotetext{
${ }^{31}$ Desde finales de los años sesenta se decía que la denominada "era audiovisual"- entonces en su momento de expansión televisiva- era la antesala de transcendentales innovaciones comunicativas que habrían de cambiar el funcionamiento de las sociedades. En esa creencia coincidían autores y organizaciones que querían cancelar el "orden mundial“" existente, como Herbert Marcuse y los movimientos de estudiantes que protagonizaron las revoluciones culturales del año 1968, con organizaciones del corte del club de Roma, que se servían de la prospectiva y del análisis de sistemas para tratar de anticipar y de reconducir los cambios a favor de la perpetración del citado orden mundial establecido.

${ }^{32}$ En aquellos años ya se habían desdibujado las utopías maltusianas de que los cambios aumentasen la libertad, la autonomía y la creatividad individuales al tiempo que fuesen disminuyendo el paro, el sobre trabajo, el hambre, la ignorancia y la explotación. Solo quedaba el eco macluhaniano del imparable advenimiento de "las nuevas tecnologías de la comunicación”, en cuyo desarrollo tenían puesta su fe tanto las derechas como las izquierdas que gobernaban en el mundo.

33 Este libro propone una teoría explicativa de cómo se va involucrando la comunicación pública en la historia. $L a$ producción social de comunicación resultará finalmente confirmada o desmentida, como todos los trabajos dialécticos, por la propia histeria de las sociedades que se encarga de verificar, de forma incuestionable, el acierto o el error de las predicciones que se hayan hecho o se deriven a partir de la teoría.
} 
- Ha sido obligado referirse como hechos observadles a algunos fenómenos comunicativos que en su momento eran sólo previsibles. Por ejemplo estaba prevista y se ha cumplido la capacidad que ahora tienen los usuarios de las tecnologías informáticocomunicativas de producir, seleccionar, modificar, difundir materiales comunicativos, Unas capacidades que hasta hace poco estaban reservadas a los mediadores profesionales y que cuando se utilicen de manera generalizada tendrán importantes consecuencias en la comunicación pública. Puede examinarse de qué manera se ha incorporado a la realidad cada predicción que se ha cumplido. Esa observación del proceso me ha proporcionado la oportunidad de fundamentar con mayor valor probatorio los contenidos del libro.

- Durante el periodo transcurrido entre la primera redacción del libro y esta tercera, he diseñado y llevado a cabo otras investigaciones de la comunicación pública que desarrollan y amplían La producción social de comunicación.( Cf. la relación que aparece en la nota) Esta nueva serie de estudios están diseñados en la mayoría de las ocasiones, para comparar dos tipos de visiones del mundo: las que se proponen en la comunicación pública y las que de hecho tienen los públicos. De este modo cabe adentrarse en los contenidos y los mecanismos de la socialización y de la influencia de los media.

La última reforma de los contenidos afecta a la bibliografía que se ha incorporado. Con ella se quieren satisfacer dos objetivos:

- El primero documental, destinado a quienes deseen conocer y ampliar en otros textos la teoría, la metodología o las fuentes de La producción social de comunicación. Es un meticuloso análisis de otras publicaciones e investigaciones mías, en el que han participado algunos de mis colaboradores y de mis editores. Los títulos que se han seleccionado fueron fundamento para este libro o me han permitido desarrollar esta última y definitiva versión. Ese trabajo documental también se refleja al hilo de la exposición, en las notas donde se va aclarando cual es la pertinencia que tienen cada una de esas obras y su contenido.

- El segundo objetivo es didáctico, para atender a quienes hacen un uso académico del libro. Esta parte de la bibliografía la he elaborado personalmente. Presenta el repertorio de las obras concretas por las que he mencionado a otros autores. Todas estas fuentes son originarias o fundacionales. Con ello quiere decirse que se ha trabajado exclusivamente con los textos de quienes crearon los temas, las ideas o los análisis que han servido de fundamento a quienes pretendemos agregar el análisis de la producción social de comunicación, al árbol de las disciplinas científicas. Los comentaristas, los divulgadores, o los saqueadores de esa herencia, no tienen lugar en este Parnaso, por muy recientes y “mediáticas” que sean sus publicaciones.

La bibliografía de los textos de otros autores, concluye con un apartado de obras que no tomé en cuenta para la redacción de este libro. Pero viene a cuento que aparezca en una bibliografía didáctica, porque en ellas se ofrecen datos, perspectivas o análisis que contribuyen a ubicar la comunicación pública, en su génesis, en su evolución o en los cambios previsibles. Como cabía esperar el repertorio de los trabajos que aportan conocimiento nuevo es limitado y no coincide necesariamente con la lista de títulos que se citan ritualmente en las bibliografías al uso. 